\title{
On Flatness and Tameness of Classes of Impartial Games
}

\author{
By
}

\author{
Tatsuya YAMADA* and Kô SAKAI ${ }^{\dagger}$
}

\begin{abstract}
For analyzing impartial games played in the misère rule, Yamasaki defined flatness of games, while Conway defined tameness. In this paper, we prove that these two concepts are equivalent.
\end{abstract}

\section{$\S 1$. Introduction}

In this paper we discuss impartial games, namely two-player games satisfying the following conditions.

- The two players move in alternate turn.

- The moves that a player can select in his turn depend not on the player but only on the position of the game. Namely, both players have the same options of moves in each position.

- Every play terminates in finitely many turns, no matter how the players move.

In what follows, we use term "game" only to mean an impartial game.

Communicated by S. Fujishige. Received September 24, 2004. Revised December 8, 2004.

2000 Mathematics Subject Classification(s): 91A46.

*Frontier Science, Graduate School of Pure and Applied Sciences, University of Tsukuba, 1-1-1, Tennoudai, Tsukuba, Ibaraki 305-8571, Japan

e-mail: ka_sui_moku_ren@yahoo.co.jp

$\dagger$ Institute of Mathematics, University of Tsukuba, 1-1-1, Tennoudai, Tsukuba, Ibaraki 305-8571, Japan

e-mail: ksakai@math.tsukuba.ac.jp

(c) 2006 Research Institute for Mathematical Sciences, Kyoto University. All rights reserved. 
In playing games, it is natural to define the last player able to move to be the winner and we call this the normal play rule. For disjunctive sums of impartial games in the normal rule, there is a well-known mathematical theory independently developed by Sprague and Grundy [3, 4].

It is possible to define the last player able to move to be the loser and we call this the misère play rule. However, the situation changes drastically. The analysis of the disjunctive sums of games in the misère rule seems much more complicated. We know very little about the strategy for playing games in the misère rule.

However, there are some types of games, for which the tactics in the misère play are obtained from those in the normal play. Conway found a type of such games and called them tame [2]. Yamasaki found another type of such games and called them flat [5].

Berlekamp, Conway, and Guy mentioned Yamasaki's work and claimed that 'his term "flat" and "projective" both imply our "tame", without any further comment [1]. In this paper, we prove that terms "tame" and "flat" are actually equivalent in spite of their apparent difference.

In Sections 2 and 3, we briefly survey general theory of impartial games. In Section 4, we prove the main result.

\section{§2. Impartial Game}

If a position $Q$ of a game is reachable from another position $P$ by a single move, $Q$ is called a successor of $P$. According to Conway [2], let us identify a position $P$ of a game with the set of all the successors of $P$. Therefore, if $P$ and $Q$ are positions of a game, $Q \in P$ means that $Q$ is a successor of $P$. All the "end-positions", which have no successors, are identified with the empty set and denoted by $\emptyset$. Conversely, any set inductively constructed from the empty set can be a position of a suitably defined game. Thus, we regard a position of any game as such a set, and vice versa.

From the above point of view, Nim-heap $* n$ for each natural number $n$ is defined inductively as follows.

Definition 2.1 (Nim heap).

$$
* 0=\emptyset, \quad * 1=\{* 0\}, \quad * 2=\{* 0, * 1\}, \ldots, * n=\{* 0, * 1, \ldots, *(n-1)\}, \ldots
$$

Definition 2.2 (sum of positions). Let $G$ and $H$ be positions of games. We define the sum $G+H$ inductively as follows:

$$
G+H=\left\{G^{\prime}+H \mid G^{\prime} \in G\right\} \cup\left\{G+H^{\prime} \mid H^{\prime} \in H\right\} .
$$




\section{$\S 3 . \quad$ Grundy Value and Winning Strategy}

It is well-known that any position of an impartial game in the normal play can be characterized completely by its Grundy value and the Grundy value of the sum of positions is equal to the Nim-sum of the Grundy values of the summands.

Definition 3.1 (Grundy value). We define the normal Grundy value $\mathcal{G}^{+}(G)$ and the misére Grundy values $\mathcal{G}^{-}(G)$ of a position $G$ inductively as follows. First, for the end-position $\emptyset$, we define

$$
\mathcal{G}^{+}(\emptyset)=0, \quad \mathcal{G}^{-}(\emptyset)=1
$$

Next, for non end-position $G$, we define

$$
\mathcal{G}^{+}(G)=\operatorname{mex}\left\{\mathcal{G}^{+}(H) \mid H \in G\right\}, \quad \mathcal{G}^{-}(G)=\operatorname{mex}\left\{\mathcal{G}^{-}(H) \mid H \in G\right\},
$$

where, for any set $S$ of ordinal numbers, we denote by mex $S$ the least ordinal number not contained in $S$. In particular, if $S \subsetneq \mathbb{N}$, mex $S$ is the least natural number not in $S$.

The following is immediate from the definitions of the Grundy values.

Lemma 3.2. Let $G$ be a position of games. Then the second player has a winning strategy at $G$ in the normal play (resp. in the misère play) if and only if $\mathcal{G}^{+}(G)=0$ (resp. $\left.\mathcal{G}^{-}(G)=0\right)$.

For any natural numbers $a$ and $b$, we denote their Nim-sum by $a \oplus b$. The Nim-sum operation is also known as the "XOR" (exclusive "OR") operation in computer science. Namely, $a \oplus b$ is the result of adding $a$ and $b$ as binary numbers without carry. It is easy to see that the Nim-sum operation can be extended naturally on the class $\mathbf{O n}$ of all ordinal numbers and $(\mathbf{O n}, \oplus, 0)$ forms a group in which every element has order 2. (To be more precise, On is a proper class in the sense of axiomatic set theory, but satisfies all the axioms for a group.)

The following is fundamental in the theory of impartial games in the normal play.

Theorem 3.3 (Grundy [3]). For any positions $G$ and $H$ of games,

$$
\mathcal{G}^{+}(G+H)=\mathcal{G}^{+}(G) \oplus \mathcal{G}^{+}(H) .
$$


However, no similar result has been obtained for general impartial games in the misère rule.

\section{§4. Analysis of Impartial Games in the Misère Rule}

Definition 4.1 (Yamasaki [5]). We define a class $\mathbb{F}$ of positions of games inductively as follows.

1. The end-position $\emptyset$ is an element of $\mathbb{F}$.

2. If $\mathcal{G}^{+}(P) \leq 1$ and a successor of $P$ is an element of $\mathbb{F}$, then $P$ is also an element of $\mathbb{F}$.

Namely, $\mathbb{F}$ is the class of all positions from which the end-position is reachable with keeping the track in positions of normal Grundy values less than 2. Moreover, we denote by $\mathbb{F}_{0}$ (resp. $\mathbb{F}_{1}$ ) the subclass of $\mathbb{F}$ consisting of all the positions of normal Grundy value 0 (resp. 1).

Clearly, $\mathbb{F}$ is the disjoint union of $\mathbb{F}_{0}$ and $\mathbb{F}_{1}$. The following is also immediate from the definition.

Lemma 4.2. If a position $G$ is not an element of $\mathbb{F}$ but at least one of its successors is in $\mathbb{F}$, then $\mathcal{G}^{+}(G)$ is greater than 1 .

The following is easily proved by induction from the definition of $\mathbb{F}$ and Theorem 3.3 .

Theorem 4.3 (Yamasaki [5]). Let $G$ and $H$ be positions of games. Then, $G+H$ is in $\mathbb{F}$ if and only if both $G$ and $H$ are in $\mathbb{F}$.

A class $\mathbb{G}$ of positions of impartial games is said to be transitive, if $P \in \mathbb{G}$ implies $P \subset \mathbb{G}$ for any position $P$. Namely, a class of positions is transitive if and only if it is closed for any moves.

Definition 4.4 (flat class). A transitive class $\mathbb{G}$ of positions is said to be flat if it satisfies the following conditions:

- For any $P \in \mathbb{G} \cap \mathbb{F}$ and any successor $Q$ of $P$, if $\mathcal{G}^{+}(Q) \leq 1$ then $Q \in \mathbb{F}$.

- For any $P \in \mathbb{G} \backslash \mathbb{F}$ and any successor $Q$ of $P$, if $Q \in \mathbb{F}$ then there exists a successor $R$ of $P$ such that $\mathcal{G}^{+}(R) \leq 1$ and satisfying either of the following conditions: 
a) $R \in \mathbb{F}$ and $\mathcal{G}^{+}(R) \oplus \mathcal{G}^{+}(Q)=1$,

b) $R \notin \mathbb{F}$ and $\mathcal{G}^{+}(R) \oplus \mathcal{G}^{+}(Q)=0$.

Definition 4.5. Let $\mathbb{G}$ and $\mathbb{G}^{\prime}$ be classes of positions of games. We define the sum class $\mathbb{G}+\mathbb{G}^{\prime}$ as follows:

$$
\mathbb{G}+\mathbb{G}^{\prime}=\left\{G+G^{\prime} \mid G \in \mathbb{G}, G^{\prime} \in \mathbb{G}^{\prime}\right\} .
$$

Clearly, if $\mathbb{G}$ and $\mathbb{G}^{\prime}$ are transitive classes, then $\mathbb{G}+\mathbb{G}^{\prime}$ is also transitive. The following two theorems are due to Yamasaki [5].

Theorem 4.6. The sum of flat transitive classes is flat.

Theorem 4.7. $\quad$ The class $\mathbb{N i m}$ of all Nim-heaps is a flat transitive class.

Yamasaki mentioned several other flat transitive classes, but we omit the details here.

Definition 4.8 (Conway [2]). Let $G$ be any impartial game and let $g=\mathcal{G}^{+}(G), \quad g_{0}=\mathcal{G}^{-}(G), \quad g_{1}=\mathcal{G}^{-}(G+* 2), \quad g_{2}=\mathcal{G}^{-}(G+* 2+* 2), \quad, \ldots$ Then, we denote the sequence $g^{g_{0} g_{1} g_{2} \ldots}$ by $\mathcal{G}_{*}(G)$. For any sufficiently large $n$ we have $g_{n+1}=g_{n} \oplus 2$, and so we write

$$
\mathcal{G}_{*}(G)=g^{g_{0} g_{1} \ldots g_{m}}
$$

if this holds for all $n$ such that $n \geq m$.

Definition 4.9 (tame class). Transitive class $\mathbb{G}$ of positions is said to be tame if $\mathcal{G}_{*}(P)$ is $0^{12}, 1^{03}$, or $n^{n}(n=0,1,2, \ldots)$ for any $P \in \mathbb{G}$.

Remark. Conway defined his "tameness" as a property of game positions [2] and generalized it later [1]. In this paper, we adopted the original and defined it as a property of classes of positions, since that treatment makes it more compatible to Yamasaki's "flatness". Yamasaki defined his "games" and "flatness" in a somewhat different framework using " $D$-scheme" and "additive class". The above definition of "flatness" is a paraphrase of a necessary and sufficient condition of his in our framework.

Theorem 4.10. $\quad$ A transitive class $\mathbb{G}$ of positions is flat if and only if any position $P \in \mathbb{G}$ satisfies the following conditions: 
(1) $\mathcal{G}^{-}(P) \oplus \mathcal{G}^{+}(P)=1$ for any $P \in \mathbb{F}$

(2) $\mathcal{G}^{-}(P) \oplus \mathcal{G}^{+}(P)=0$ for any $P \notin \mathbb{F}$.

Proof. First we prove the necessity of conditions (1) and (2). Let $\mathbb{G}$ be flat. We prove the claims by induction on the structure of position $P$. If $P=\emptyset$, then $P \in \mathbb{F}$ and $\mathcal{G}^{-}(P) \oplus \mathcal{G}^{+}(P)=1 \oplus 0=1$. Let $P \neq \emptyset$. We have three cases.

Case 1. If $P \in \mathbb{F}_{1}$, we have to prove that $\mathcal{G}^{-}(P)=0$, for which it is sufficient to prove that there is no successor $Q$ of $P$ such that $\mathcal{G}^{-}(Q)=0$. If such a successor $Q$ exists, then the induction hypothesis assures that

(a) if $Q \in \mathbb{F}$ then $\mathcal{G}^{+}(Q)=1=\mathcal{G}^{+}(P)$, and

(b) if $Q \notin \mathbb{F}$ then $\mathcal{G}^{+}(Q)=0$.

However, (a) contradicts the definition of the Grundy value, and (b) contradicts the flatness of $\mathbb{G}$.

Case 2. If $P \in \mathbb{F}_{0}$, we have to prove that $\mathcal{G}^{-}(P)=1$. By the definition of $\mathbb{F}_{0}, P$ must have a successor $Q$ in $\mathbb{F}_{1}$, and the induction hypothesis said that $\mathcal{G}^{-}(Q)=0$. Similar discussion as in Case 1 proves that there is no successor $Q$ of $P$ such that $\mathcal{G}^{-}(Q)=1$. Therefore, we have $\mathcal{G}^{-}(P)=1$.

Case 3. If $P \notin \mathbb{F}$, there are four subcases.

Case 3-1. If $P$ has successors $Q \in \mathbb{F}_{0}$ and $R \in \mathbb{F}_{1}$, then $\mathcal{G}^{-}(Q)=1$ and $\mathcal{G}^{-}(R)=0$ by the induction hypothesis. Since $\mathcal{G}^{+}(S)=\mathcal{G}^{-}(S)$ for any successor $S \notin \mathbb{F}$ by the induction hypothesis, we can conclude that $\mathcal{G}^{+}(P)=\mathcal{G}^{-}(P)$.

Case 3-2. If $P$ has a successor $Q \in \mathbb{F}_{1}$ but has no successor in $\mathbb{F}_{0}$, then there exists a successor $R$ of $P$ such that $R \notin \mathbb{F}$ and $\mathcal{G}^{+}(R)=1$ by the flatness condition. The induction hypothesis assures that $\mathcal{G}^{-}(Q)=0$ and $\mathcal{G}^{-}(R)=1$. Therefore, we have $\mathcal{G}^{-}(P) \geq 2$. Since $\mathcal{G}^{+}(P)$ exceeds 1 by Lemma 4.2, we can conclude that $\mathcal{G}^{+}(P)=\mathcal{G}^{-}(P)$ by the induction hypothesis on the other successors of $P$.

Case 3-3. If $P$ has a successor $Q \in \mathbb{F}_{0}$ but has no successor in $\mathbb{F}_{1}$, a similar discussion as in Case 3-2 proves that $\mathcal{G}^{+}(P)=\mathcal{G}^{-}(P)$.

Case 3-4. If $P$ has no successors in $\mathbb{F}$, then the induction hypothesis easily proves that $\mathcal{G}^{+}(P)=\mathcal{G}^{-}(P)$.

Next, we prove the sufficiency of the conditions. Let $\mathbb{G}$ be a transitive class satisfying conditions (1) and (2). 
Assume that a position $P \in \mathbb{G} \cap \mathbb{F}$ has a successor $Q$ such that $\mathcal{G}^{+}(Q) \leq 1$. If $Q \notin \mathbb{F}$, we have $\mathcal{G}^{+}(Q)=\mathcal{G}^{-}(Q) \leq 1$ and $\mathcal{G}^{+}(P) \oplus \mathcal{G}^{-}(P)=1$ by condition (1). However, since $\mathcal{G}^{+}(P) \leq 1$, this implies $\mathcal{G}^{+}(P)=\mathcal{G}^{+}(Q)$ or $\mathcal{G}^{-}(P)=$ $\mathcal{G}^{-}(Q)$, which contradicts the definition of the Grundy values. This proves the first condition of the flatness.

Assume that a position $P \in \mathbb{G} \backslash \mathbb{F}$ has a successor $Q \in \mathbb{F}$. By Lemma 4.2, $\mathcal{G}^{+}(P)$ is greater than 1. Moreover, condition (2) assures that $\mathcal{G}^{+}(P)=\mathcal{G}^{-}(P)$, and hence there exists a successor $R$ of $P$ such that $\mathcal{G}^{-}(Q) \oplus \mathcal{G}^{-}(R)=1$. Since $\mathcal{G}^{-}(Q) \oplus \mathcal{G}^{+}(Q)=1$ by condition $(1)$, we have $\mathcal{G}^{-}(R) \oplus \mathcal{G}^{+}(Q)=0$. Again by condition $(1), \mathcal{G}^{-}(R) \oplus \mathcal{G}^{+}(R)=1$ if $R \in \mathbb{F}$, but $\mathcal{G}^{-}(R) \oplus \mathcal{G}^{+}(R)=0$ if $R \notin \mathbb{F}$. Therefore, in either case, we can conclude that position $R$ has the property required by the second condition of the flatness.

Corollary 4.11. A transitive class $\mathbb{G}$ is flat if and only if it is tame.

Proof. Let $\mathbb{G}$ be tame. We prove that $\mathbb{G}$ satisfies conditions (1) and (2) of Theorem 4.10. It is sufficient to show that

$P \in \mathbb{F}_{0}$ if $\mathcal{G}_{*}(P)=0^{12}, \quad P \in \mathbb{F}_{1}$ if $\mathcal{G}_{*}(P)=1^{03}, \quad$ and $\quad P \notin \mathbb{F}$ if $\mathcal{G}_{*}(P)=n^{n}$, since no other $\mathcal{G}_{*}$-values are possible. We prove this by induction on the structure of $P$. If $P=\emptyset$, then $\mathcal{G}_{*}(P)=0^{12}$ and $P \in \mathbb{F}_{0}$ by the definition. Let $P \neq \emptyset$. If $\mathcal{G}_{*}(P)=0^{12}$, then $P$ must have a successor $Q$ such that $\mathcal{G}^{-}(Q)=0$ by the definition of the Grundy value. But, $\mathcal{G}^{+}(Q)$ cannot be equal to 0 since $\mathcal{G}^{+}(P)=0$. Therefore, the only possible $\mathcal{G}_{*}$-value of $Q$ is $1^{03}$ and $Q \in \mathbb{F}_{1}$ by the induction hypothesis. Thus, $P \in \mathbb{F}_{0}$ by the definition. Similarly, $P$ is in $\mathbb{F}_{1}$ if $\mathcal{G}_{*}(P)=1^{03}$. If $\mathcal{G}_{*}(P)=n^{n}$ such that $n \geq 2$, then $P \notin \mathbb{F}$ clearly. If $\mathcal{G}_{*}(P)=1^{1}$ or $\mathcal{G}_{*}(P)=0^{0}$, then $P$ cannot have a successor of $\mathcal{G}_{*}$-value $0^{12}$ or $1^{03}$. Thus, we conclude that $P \notin \mathbb{F}$ in this case by the induction hypothesis.

Conversely, let $\mathbb{G}$ be flat. Since $* 2 \notin \mathbb{F}$, Theorem 4.3 guarantees that there is no position $P$ such that $P+* 2+\cdots+* 2 \in \mathbb{F}$. Therefore, since the sum $\mathbb{G}+\mathbb{N i m}+\cdots+\mathbb{N i m}$ is a flat transitive class by Theorems 4.6 and 4.7 ,

$$
\mathcal{G}^{-}(P+* 2+\cdots+* 2)=\mathcal{G}^{+}(P+* 2+\cdots+* 2)=\mathcal{G}^{+}(P) \oplus 2 \oplus \cdots \oplus 2
$$

holds for any position $P \in \mathbb{G}$ by condition (2) of Theorem 4.10. Now, the following are straightforward conclusions of conditions (1) and (2) of Theorem 4.10 .

$$
\begin{aligned}
& \cdot \mathcal{G}_{*}(P)=0^{12} \text { if } P \in \mathbb{F}_{0}, \\
& \cdot \mathcal{G}_{*}(P)=1^{03} \text { if } P \in \mathbb{F}_{1},
\end{aligned}
$$


. $\mathcal{G}_{*}(P)=n^{n}$ if $P \notin \mathbb{F}$, where $n=\mathcal{G}^{+}(P)=\mathcal{G}^{-}(P)$.

It is clear from the definition and Lemma 3.2 that, for positions in a tame (or equivalently flat) class, there is a winning strategy based on the Grundy values both in the misère and the normal rules. Moreover, Theorem 4.6 assures that such a strategy can be easily extended to positions of the sum of tame (or flat) classes.

\section{References}

[1] Berlekamp, E. R., Conway J. H. and Guy, R. K., Winning Ways for your mathematical plays, volume 2 (second edition), A. K. Peters, 2003.

[2] Conway, J. H., On Numbers and Games (second edition), A. K. Peters, 2001.

[3] Grundy, P. M., Mathematics and games, Eureka, 2 (1939), 6-8.

[4] Sprague, R. P., Über mathematische Kampfspiele, Tôhoku Math. J., 41 (1935-6), 291-301.

[5] Yamasaki, Y., On misère Nim-type games, J. Math. Soc. Japan, 32 (1980), 461-475

[6] Yamada, T. and Sakai, K., On flatness of impartial games (in Japanese), IPSJ SIG Technical Report, 2004-GI-11, 89-94. 